\title{
Metrological atomic force microscope and traceable measurement of nano-dimension structures
}

\author{
Sitian Gao, Mingzhen Lu, Wei Li, Yushu Shi, Qi Li \\ National Institute of Metrology, 100013, Beijing, China
}

ABSTRACT

The quantity assurance in semiconductor industry development requires dimensional measurements with nanometer accuracy. A metrological AFM is designed to establish a traceable standard with nanometer uncertainty. The principle and design of the instrument are introduced in this paper. The displacement of the sample is traced to the SI unit by interferometers. The metrological AFM is applied to step height and line pitch measurements. The results are compared with an optical instrument and a profilometer. The metrological AFM is used for step height measurement in an international comparison and the result shows an uncertainty less than $2 \mathrm{~nm}$. The application of the metrological AFM in pitch measurements is also introduced.

Keywords: nanometrology; AFM; traceability; nanostructure; pitch; linewidth; step height;

Citation: Sitian Gao, Mingzhen Lu, Wei Li, Yushu Shi, Qi Li, "Metrological atomic force microscope and traceable measurement of nano-dimension structures", Acta IMEKO, vol. 2, no. 1, article 6, August 2013, identifier: IMEKO-ACTA-02(2013)-01-06

Editors: Paolo Carbone, University of Perugia, Italy; Ján Šaliga, Technical University of Košice, Slovakia; Dušan Agrež, University of Ljubljana, Slovenia Received January $10^{\text {th }}, 2013$; In final form July 11 ${ }^{\text {th }}, 2013$; Published August 2013

Copyright: (C) 2013 IMEKO. This is an open-access article distributed under the terms of the Creative Commons Attribution 3.0 License, which permits unrestricted use, distribution, and reproduction in any medium, provided the original author and source are credited

Funding: none reported

Corresponding author: Sitian Gao, e-mail: gaost@nim.ac.cn

\section{INTRODUCTION}

With the development of semiconductor industry, the line width of integrated circuits is decreasing to tens of nanometers. The miniaturization in semiconductor manufacturing requires measurements with nanometer accuracy in the lithographic process. Accurate dimensions and position of patterns are essential for present industrial quality.

The main industrial countries around the world have been working at establishing metrological specifications and instruments relevant to nanotechnology. The Discussion Group 7 (DG7) for nanometrology under the Consultative Committee for Length's Working Group on Dimensional Metrology (CCLWGDM) decided to perform a comparison for five different types of artifacts in order to set up an international nanometrological regime.

Atomic force microscopes (AFMs) provide high resolution and quantitative measurements for nano- and micro-scaled structures [1]. AFMs are engaged in dimensional measurements including distance, pitch, width, diameter, geometry, roughness, and thickness [2]. The probe or the sample is usually scanned by a piezotube and the displacement is measured by capacitive sensors. Due to the nonlinearity and hysteresis of the piezo scanner, AFMs with metrological abilities are required for consistency in the measurement quality.

Most metrological AFMs integrate laser interferometers to ensure direct traceability to the SI unit of length, and have a full evaluation of uncertainty budget to assign an uncertainty to the measurement results. AFMs can be calibrated by using transfer standards such as step height, 1D or 2D gratings and 3D pyramids, which are well characterized and traced to metrological AFMs.

At the National Institute of Metrology (NIM), a metrological AFM has been developed and improved. In this paper, we report the principle and architecture of the metrological AFM. Then the calibration applications of the metrological AFM in step height and pitch artefacts are demonstrated.

\section{METROLOGICAL AFM}

\subsection{Principle of AFM}

AFM is based on the interaction between a sharp tip and samples. When the cantilever is approaching the sample surface, the interaction between the tip and sample will cause bending of the cantilever. The method of cantilever sensing 


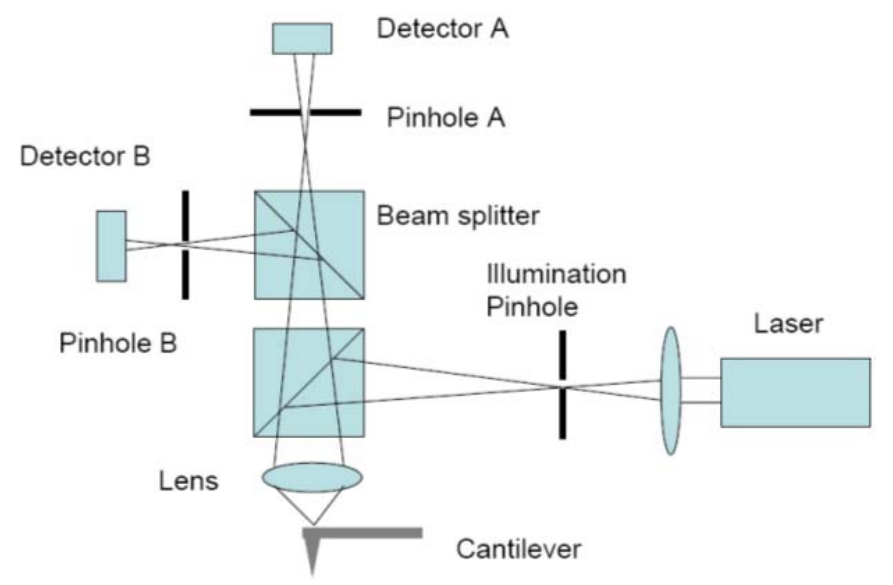

Figure 1. AFM with confocal sensing technique.

here is a confocal method different from conventional quadrant photodiode detection. The confocal detection principle of the AFM VERITEKT (Zeiss) is illustrated in Figure 1.

The laser beam through the pinhole is focused onto the cantilever and reflected to the beam splitter. Then the beam is separated and focused to the two detectors. The two pinholes before the detectors are arranged before and after the focal spots respectively. When the interaction between the tip and sample causes bending of the cantilever, the two detectors have opposite responses to the displacement of the cantilever tip. The reflected laser beam and the incident beam share the same optical path and the structure is stable, so the signal is less disturbed by air. The cantilever is $100 \mu \mathrm{m}$ in length and the tip height is $10-15 \mu \mathrm{m}$ with radius less than $10 \mathrm{~nm}$.

To reduce the fluctuation of the laser intensity, the difference of the signals is divided by the summation. The signal shows a linear relation to the displacement of the sample to tip as shown in Figure 2. The signal is detected for feedback control of the piezostage, to keep the distance between the tip and sample constant. So the sensor serves as the zero point indicator.

\subsection{Metrological AFM instrument}

In the commercial AFM using a piezotube to drive the probe, the bending of the tube will change the length of the tube in $\mathrm{z}$-direction when scanning in the xy plane [3]. The scanning surface is curved and requires calibration by standards.

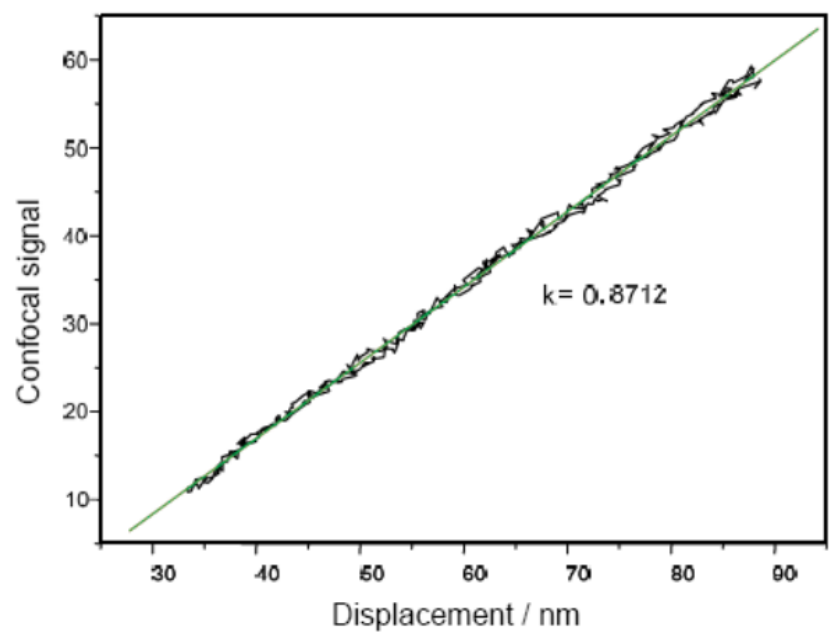

Figure 2 . The cantilever signal versus the tip displacement.
Y Interferometer

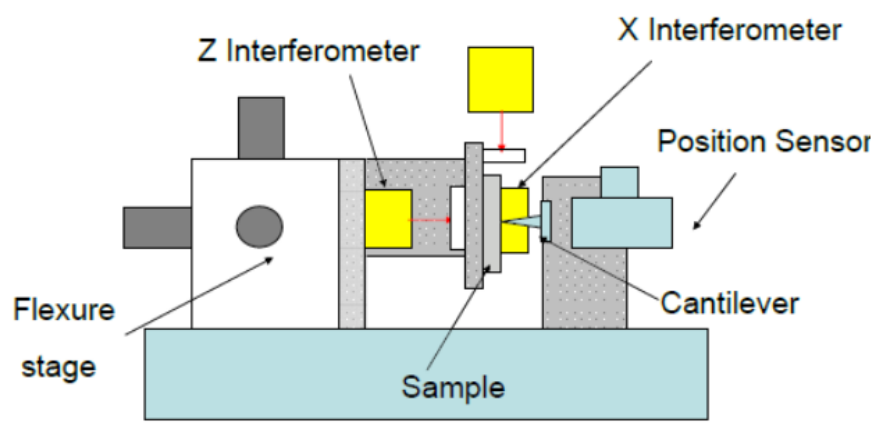

Figure 3. Structure of metrological AFM.

The displacements also require calibration for the nonlinearity of the piezoelectric actuators.

Most of the metrological AFMs are equipped with interferometers to calibrate the position and trace the displacement to SI unit [4]. By the incorporation of three miniature laser interferometers, an AFM (Zeiss) has been modified to the metrological AFM in cooperation with the Ilmenau Technical University and the Physikalisch-Technische Bundesanstalt [5].

The design is shown in Figure 3. The sample stage is moved by a 3-dimensional scanner. The monolithic flexure hinge stage is driven by three linear piezoceramic elements with capacitive sensors in three directions. The flexure hinge is designed with different lengths and deflection angles to achieve a scanning range of $70 \mu \mathrm{m} \times 15 \mu \mathrm{m} \times 15 \mu \mathrm{m}$. The displacements in three axes are traceable by interferometers with a $633 \mathrm{~nm}$ laser. The maximum angular motion of the stage is 0.5 arcsec. So the tip of the AFM is placed at the intersection of the interferometer beams, to eliminate the Abbe error.

The displacement signal is sent by a computer to the $\mathrm{D} / \mathrm{A}$ converter to drive the piezostage. The difference between the expected position $\mathrm{P}$ and the real position $\mathrm{P}_{\mathrm{s}}$ measured by the interferometers is caused by the motion coupling between different axes and the non-linearity error of the motion axes. The compensation function is derived from the measurement.

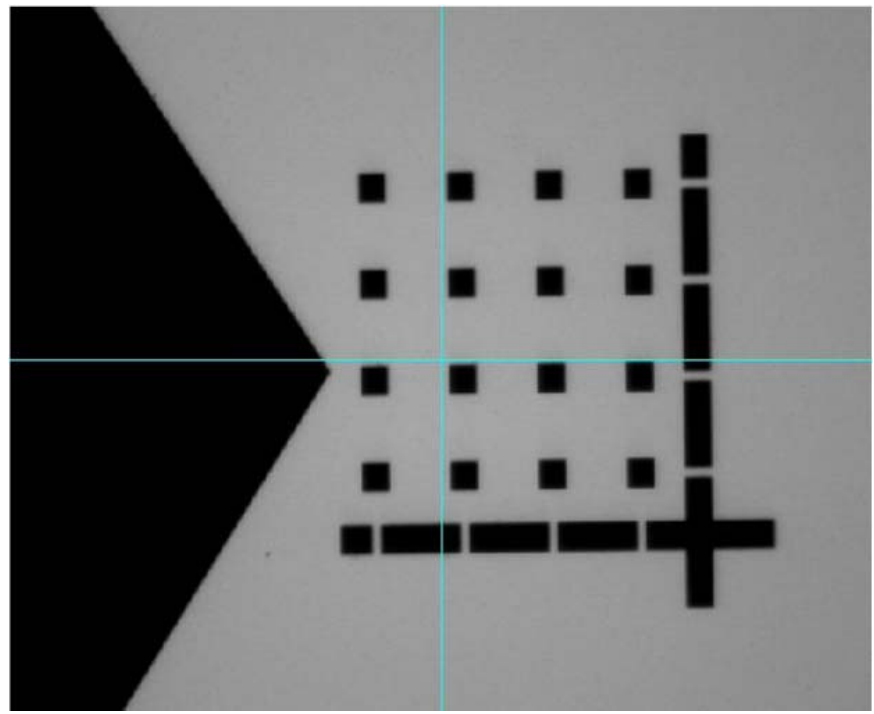

Figure 4. A sample of a step height standard. 
Table 1. Step height standard results measured with different instruments (nm).

\begin{tabular}{cccc}
\hline No & Profilometer $^{\mathrm{a}}$ & Metrological AFM & AFM (PTB) $^{\mathrm{b}}$ \\
\hline 1 & 301.0 & 300.5 & 301.3 \\
2 & 396.0 & 393.5 & 391.7 \\
3 & 593.5 & 598.9 & 595.6 \\
4 & 924.0 & 922.7 & 923.6 \\
5 & 1079.0 & 1077.0 & 1075.1 \\
6 & 1739.0 & 1730.0 & 1722.7 \\
\hline
\end{tabular}

\section{a. Alpha step 200}

b. Metrological SPM with interferometer to calibrate

\section{MEASUREMENT RESULTS}

The step height, line width and pitch are three important parameters of nano-structure dimensions in integrated circuit manufacturing. To provide consistent and traceable measurements, the transfer standards are calibrated by the metrological AFM and then are used to calibrate the instrument in industry and research.

\subsection{Step height}

Step height standards are utilized to calibrate the z-axis of microscopes and topography measuring instruments. A step sample is shown in Figure 4. It is fabricated on a $\mathrm{Si}$ substrate with $\mathrm{SiO}_{2}$ step squares and bars. Six height samples are fabricated with nominal heights $300 \mathrm{~nm} ; 400 \mathrm{~nm} ; 600 \mathrm{~nm} ; 900$ $\mathrm{nm} ; 1000 \mathrm{~nm}$; and $1800 \mathrm{~nm}$. The samples are measured with a profilometer and an AFM to compare different instruments and methods. The results are shown in Table 1 . The step height results measured with different instruments show consistent results with deviations less than $10 \mathrm{~nm}$.

To establish nanometrological equivalence in the nanoscale regime, international comparison of nano-standards started from the year 2000. Step height international comparisons with PTB as the pilot laboratory has been accomplished [6]. The standards are $\mathrm{Si}$ substrates with $\mathrm{SiO}_{2}$ steps coated with $\mathrm{Cr}$. The measured region is $100 \mu \mathrm{m} \times 100 \mu \mathrm{m}$.

The scanning area of the instrument is $70 \mu \mathrm{m} \times 12 \mu \mathrm{m}$ with $400 \times 200$ pixels, so three subregions on the standard are scanned from top down in the $100 \mathrm{~nm}$ range. The result of a 20 $\mathrm{nm}$ step height sample is shown in Figure 5. For each profile, the $15 \mu \mathrm{m}$ lines marked on the upper step profile and the lower surface are fitted with a least square criterion to obtain the step height. The line step heights at different positions are averaged.

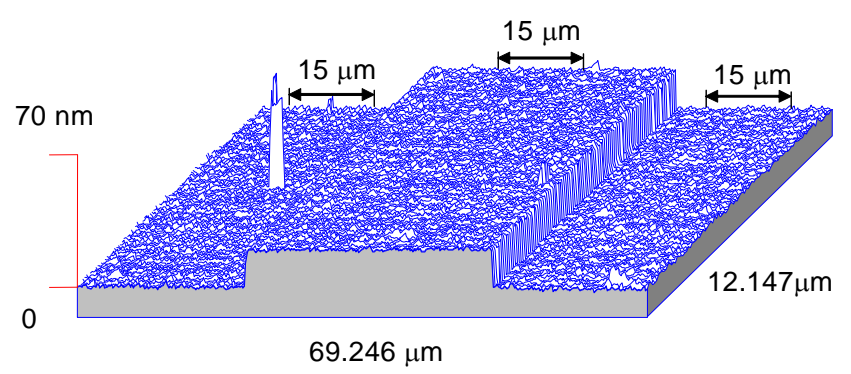

Figure 5. Image of a $20 \mathrm{~nm}$ step sample measured by the metrological AFM.

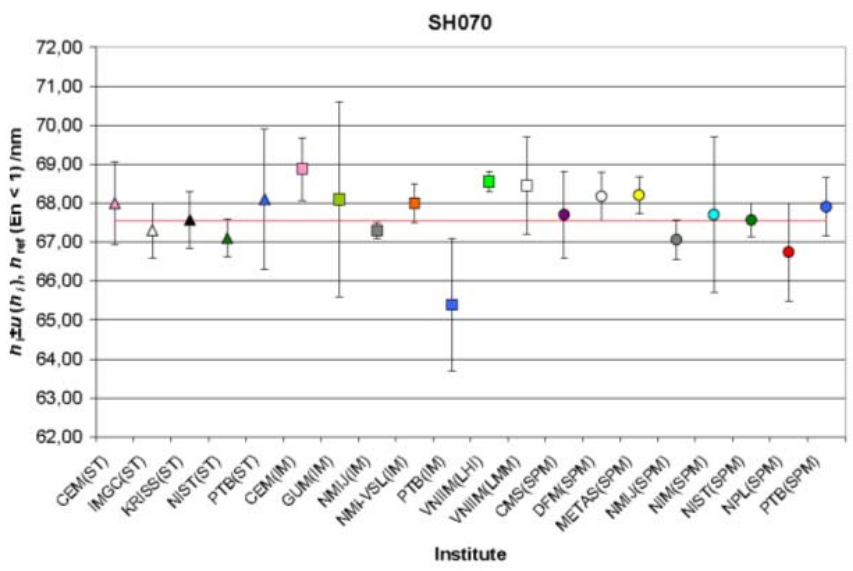

Figure 6. Measured step heights $h_{\mathrm{i}}$ of the participating institutes and reference value $h_{\text {ref }}$ (red line) of the $70 \mathrm{~nm}$ step height sample.[6]

Fifteen national metrology institutes joined the comparison with different instruments. The result of the $70 \mathrm{~nm}$ step height is shown in Figure 6. The uncertainty of the measured result of NIM is no more than $2 \mathrm{~nm}$. The uncertainty is somewhat larger compared with others. In fact the crosstalk in the z-direction caused by movement in the $x^{-}$and $y$-axes is the main uncertainty component in the step height measurement and this is independent of the step height. The uncertainty for large step height measurement results are relatively small compared with those of the other participant [6].

\subsection{D Grating pitch}

The lateral magnification of the AFM can be calibrated by $1 \mathrm{D}$ or $2 \mathrm{D}$ pitch standards. These standards are calibrated by the metrological AFM. For the periodic structure, the gravity centres of the grating structures are calculated and then the position of the centres are averaged to obtain the pitch value. The results of a $3 \mu \mathrm{m}$ pitch are shown in Figure 7 . The

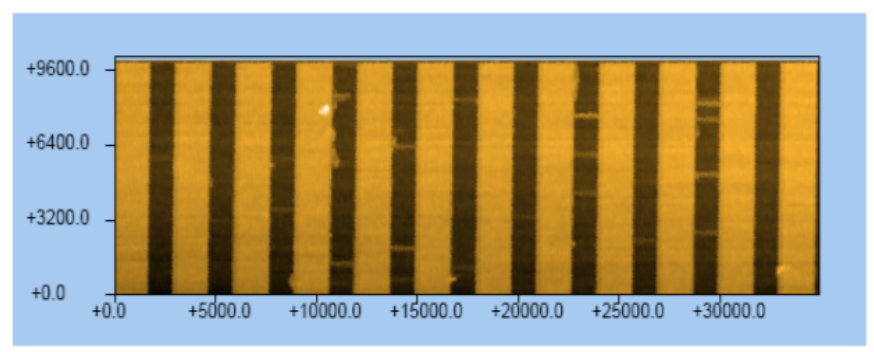

(a)

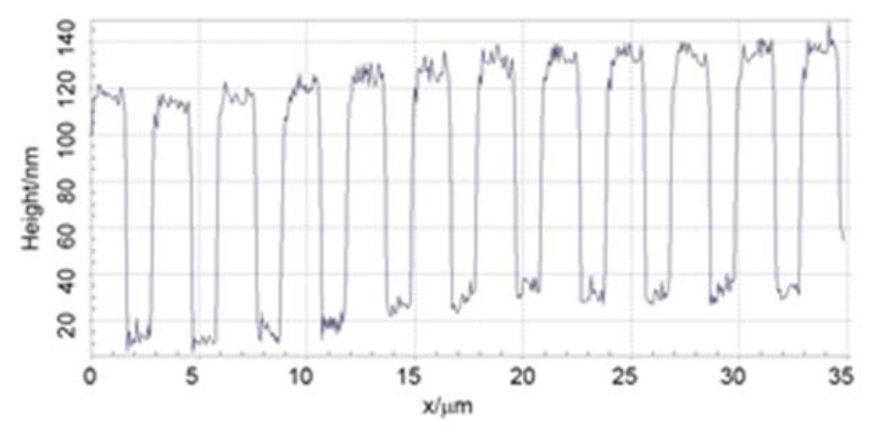

(b)

Figure 7. Measurement results of a $3 \mu \mathrm{m} 1 \mathrm{D}$ grating with $35 \mu \mathrm{m} \times 10 \mu \mathrm{m}$ scanning range (a) and a 1D scanning profile of the grating (b). 


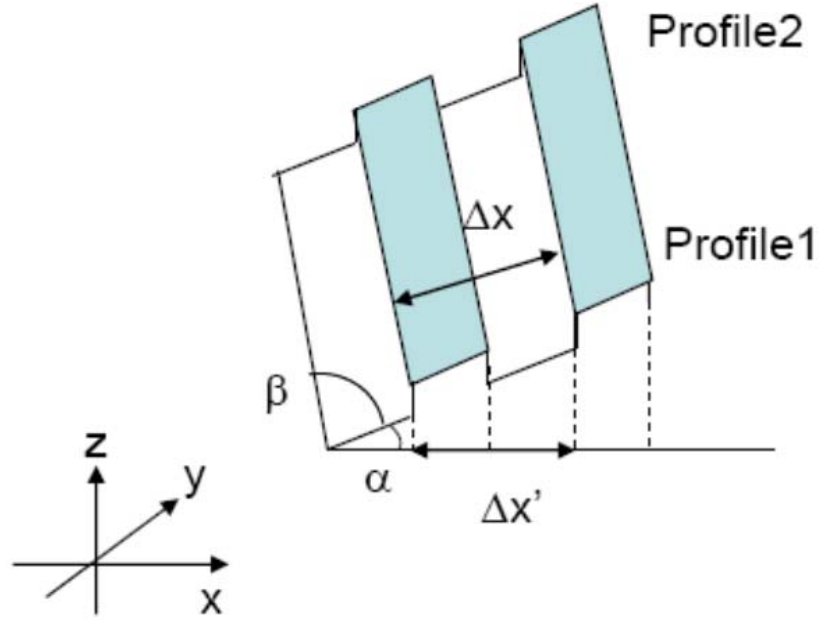

Figure 8. Error correction of the sample incline.

scanning range is $35 \mu \mathrm{m} \times 10 \mu \mathrm{m}$. The profile is shown in Figure 7 (b). The height of the grating is about $110 \mathrm{~nm}$.

A threshold line across the profile is chosen to separate the grating structures. The gravity centre of each structure is calculated and the average pitch is determined [7]. Usually the sample is mounted with an angle deviation relative to the interferometers axes. The incline of the sample causes a cosine error. As shown in Figure 8, the error from the angle between the profile and the $\mathrm{x}$-axis $\alpha$ can be corrected by fitting the baseline of the single profile. Also the sample orientation in the sample plane relative to the interferometers axes is corrected. A series of measurements along the $y$-axis is required to calculate the orientation of the grating bars. The real pitch is obtained by

$$
\Delta x=\frac{\Delta x^{\prime} \sin (\beta)}{\cos (\alpha)},
$$

where $\beta$ is the angle between the measured profile and the direction of the bars.

The average pitch is $3.01 \mu \mathrm{m}$ with a standard deviation of $0.05 \mu \mathrm{m}$ due to the inhomogeneity of the sample. The uncertainties of the wavelength and the temperature are negligible. The uncertainty of the instrument due to the coupling of the different axes and the nonlinearity is $1.15 \mathrm{~nm}$. The uncertainty due to the cosine error caused by the sample mounting after correction is $0.03 \mathrm{~nm}$.

Because the probe tip of the AFM has a finite size, the measured profile of the step edge is the convolution of the tip shape and the real step profile. This edge effect is not significant for step heights or line pitch measurements of the periodic structure, for the edges have the same influence and can be eliminated.

However the line width is influenced by the tip size of the AFM probe. So the definition of the line width is under study to retrieve the real width.

\section{CONCLUSIONS}

A metrological AFM is designed to establish a traceable standard with nanometer uncertainty. The sample stage is driven by a piezostage and reflectors are mounted on the stage as reference mirrors of interferometers, so that the movements of the stage in 3 directions are traceable.

For step height measurements, the metrological AFM gives comparable results with other instruments and the AFM demonstrates an uncertainty $\mathrm{U}_{95}<2 \mathrm{~nm}$. For the pitch measurement, the uncertainty from the instrument is less than 2 $\mathrm{nm}$ which is mainly due to the coupling of the axes and the sample incline.

The scanning range limits the application of the metrological AFM. A large range metrological AFM has been developed at the NIM to calibrate larger samples [8].

\section{REFERENCES}

[1] A.Yacoot, L. Koenders, "Recent developments in dimensional nanometrology using AFMs", Meas. Sci. Technol., 22, (2011), pp. 122001.

[2] J. A. Kramar, R. Dixson, N. G. Orji, "Scanning Probe Microscope Dimensional Metrology at NIST", Meas. Sci. Technol. 22, (2011), pp. 024001.

[3] J. Kwon, J. Hong, Y. Kim, "Atomic force microscope with improved scan accuracy, scan speed and optical vision”, Rev Sci. Instrum., 74, (2003), pp. 4378-4383.

[4] G. Dai, F. Pohlenz, H. Danzebrink, K. Hasche, G. Wilkening, "Improving the performance of interferometers in metrological scanning probe microscopes", Meas. Sci. Technol. 15, (2004), pp. 444-450.

[5] M. Bienias, S. Gao, K. Hasche, R. Seemann, K. Thiele, “A metrological scanning force microscope used for coating thickness and other topographical measurements", Applied Physics A., 66, (1998), pp. S837-S842.

[6] L. Koenders. "WGDM-7: Preliminary Comparison on Nanometrology According to the rules of CCL key comparisons NANO 2 Step height Standards", PTB Braunschweig, (2003).

[7] G. Dai, L. Koenders, F. Pohlenz, T. Dziomba, H. Danzebrink, "Accurate and traceable calibration of one-dimensional gratings", Meas. Sci. Technol. 16, (2005), pp. 1241-1249.

[8] M. Lu, S. Gao, Q. Li, W. Li, Y. Shi, X. Tao, "Long range metrological atomic force microscope with versatile measuring head", Proc. SPIE 8759, Eighth International Symposium on Precision Engineering Measurement and Instrumentation, 2012, 8-11, August, China, pp. 87594Y. 